\title{
Blockchain Approach for Smart Health Wallet
}

\author{
Meetkumar Patel ${ }^{1}$ \\ Student, Department of Computer Engineering, Ahmedabad Institute of Technology, Ahmedabad, India ${ }^{1}$
}

\begin{abstract}
Health being a very important issue nowadays, a major problem facing health care systems throughout the world is how to share medical records/data with more collaborator for more purposes, and we also ensure about data integrity and protecting patient privacy. With our study hospitals and the clinic will be able to give all control of medical records to patients and increasing accessibility of medical records and also enhancing the security of records through the power of Blockchain and cloud structure. Each and every patient will have its own secure manageable cloud space. Patients can store and manage medical records in their cloud health wallet anytime anywhere. The patient can replace hardcopy of medical records with their cloud medical wallet.
\end{abstract}

Keywords: Blockchain, Health Wallet, Medical Record, Cryptography.

\section{INTRODUCTION}

The entire world is racing speedily towards digitization and The evolution of computers, especially the internet has affected to manually record management systems. Many years ago, we had been investing our time to manage patient records and information manually But nowadays management systems are available for hospital and as well as other sectors.

The aim of any Records Management System is the right information in the right place in the right order, at the right time for the right person at the lowest cost. But there are many hospital management and EHR [Electronic Health record] systems available in the market. EHRs were never designed to manage multi-institutional, lifetime medical records. Interoperability challenge between different providers and hospital systems pose another barrier to effective data sharing. This lack of coordinated data management and exchange means health records are fragmented, rather than cohesive [1]

Medical records/data also played an important role for research. The ONC's report emphasizes that biomedical and health researchers "require the ability to analyze information from many sources in order to identify public health risks, develop new treatments and cures, and enable precision medicine" [2]. These medical records are beneficial for medical researchers, clinical studies, surveys and educational level. The greatest problem we came across was one of security. In our study, we explore a blockchain structure applied to the medical records. We build on this distributed ledger protocol originally associated with Bit-coin[3]. Basically, the blockchain uses public key cryptography. Copies of the blockchain are distributed on each and every participating node in the network.

Our Secure Health wallet blockchain implementation addresses the four issues highlighted: fragmented, slow access, system interoperability, improve data quality and quantity for medical research as well as patients. Health wallet is also providing facilities directly or indirectly in different sectors like pharmacy, medical research, government, insurance and etc.

\section{RELATED WORK}

[4] According to Gustavo H. M. B. Motta and Sergio S. the design of proper models for authorization and access control for the electronic patient record is essential to a wild scale use of ERP in a large health organization. They also propose a contextual role-based access control authorization model aiming to increase the patient privacy and the confidentiality of patient data, whereas being flexible enough to consider specific cases. They propose in their paper a contextual role-based access control (RBAC) authorization model for the ERP that extends the proposed National Institute of Sanders and Technology (NIST) RBAC reference model.

MIT Graduate student researchers Ariel Ekblaw, Asaf Azaria and Thiago Vieira, along with Senior Research Scientist Andrew Lippman, are developing a cryptocurrency-backed technology for electronic healthcare records They are developing a system, called MedRec[5], for managing medical records that use the Ethereum blockchain[6].

Hyperledger is an umbrella project of open source blockchains and related tools, started in December 2015 by the Linux Foundation, to support the collaborative development of blockchain-based distributed ledgers[7]. Hyperledger blockchain provides various types of platforms like Hyperledger Burrow, Hyperledger Fabric, Hyperledger Iroha and Hyperledger Sawtooth. Hyperledger Fabric is a permission blockchain infrastructure, originally contributed by IBM and Digital Asset [8]. 
Blockchain Health Co. is a San Francisco-predicated software company that utilizes blockchain technology to engender a direct connection between medical research and users. BHC allows utilizers to apportion information directly to researchers utilizing their platform built on the blockchain[9].

\section{METHODOLOGY}

The Smart Health Wallet can provide patients with access to and control over their medical information and medical record. Blockchain approach provides a vehicle to facilitate information sharing between patients and their providers. Medical data can be stored in the blockchain using several approaches. The smart health wallet methodology consists of two main approaches. In the first approach, provider gives significant access to their database using our smart health wallet API and in the second approach, provider access our smart panel to enter patient database using Qr code which one generated by the patient. Here the providers are doctors, chemists, hospitals, labs and etc. Here in the following figure, we can see the architecture of the SHW (Smart Health Wallet).

\section{An Architecture of SHW (Smart Health Wallet):}

In architecture, we can see that providers can store patient's data in blockchain using our smart health wallet API. Here the hyperledger provides the frameworks for blockchain. Once the patient data is written to the blockchain, it can only be decrypted using patient's private key, thus providing patients with control over information transferred between provider organizations. Blockchain technology supports the use of smart contracts. Smart contracts allow us to automate and track certain state transitions. The smart contract could check to ascertain that the blockchain was being updated with medical information from that provider, thereby providing the patients with an automated way to ascertain information is made available to other provider organization.

When providers need access to the patient's medical records/information in the blockchain, the provider's system could automatically contact the blockchain where the patient has granted access. Authorized providers could obtain access in accordance with the patient's preferences and patients would also know which providers were accessing their medical information. Patients could grant full access or choose to limited access to some information based upon preferences.

Here in the following figure, we can see the architecture of the SHW (Smart Health Wallet).

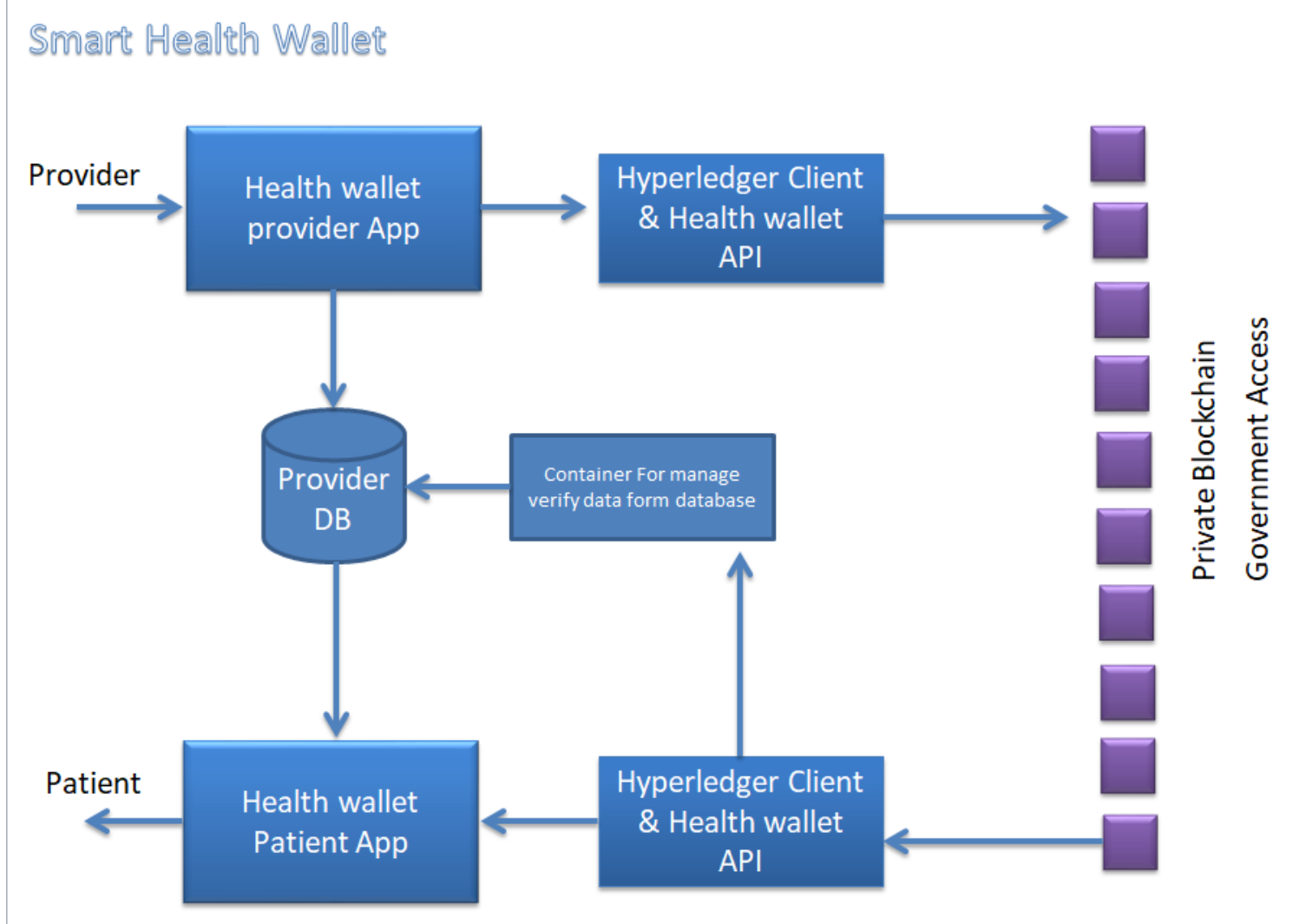

Fig 1 Architecture of the SHW (Smart Health Wallet) 


\section{B. API Access Approach:}

In API access approach provider can update patient's information through the smart health API. Smart health API directly connect with the provider's database. The connection was made by using the authentication layer. Authentication layer can authenticate both sides. The provider can also set their limitation to access their database. If the patients did not grant permission to update or access their medical records then provider did not make any change or access the patient's medical records. API layered approach consists four layers.

- Provider DB: In this layer provider firstly grant access to API. And provider can set limitation to access their database by API. The provider also knows that which data are accessed by API. If the provider makes changes on their database then automatically updated on blockchain database through API.

- Authentication Module: Authentication module firstly authenticates each and every requisite which one made by provider or API. Firstly its check and validate the API key of the provider and their limitations. And then check the provider request was fulfill by patients criteria means patients have grant access or not.

- Smart health module API: Smart health module API is making a bridge between provider database and blockchain database. It was operated by API key.

- Blockchain structure: This all data are store in blockchain structure. Blockchain structure uses decentralized database concept.

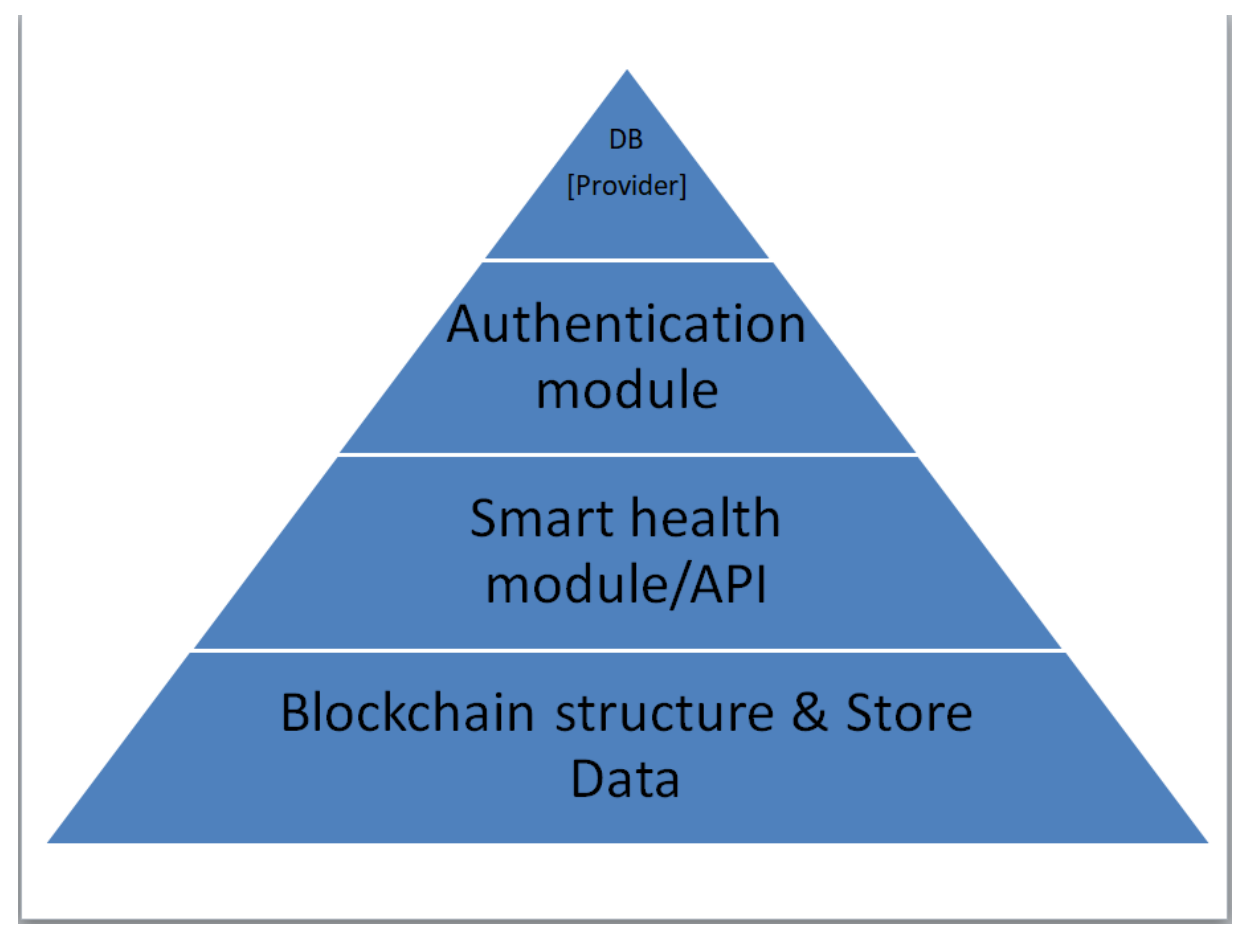

Fig 2 API layered approach

\section{Smart Portal Access:}

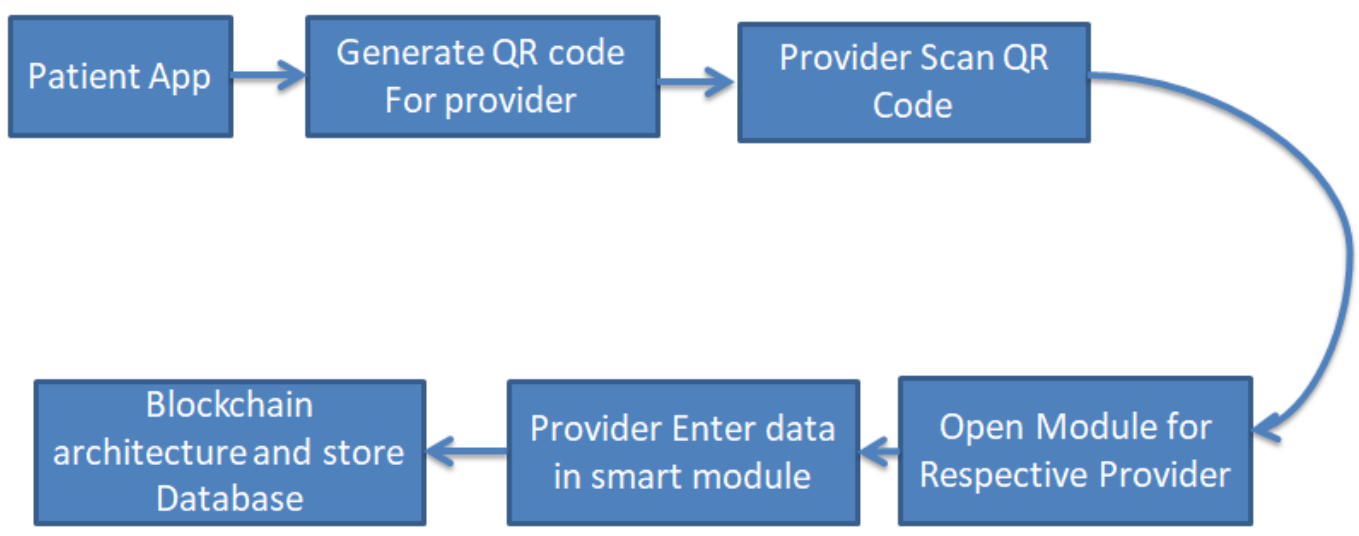

Fig 3 Smart portal Access 
This approach was useful when the provider was not convinced to share their database then this approach gave mobility for the patient. Here patient can generate QR code respective their providers. Provider scan this QR code and then automatically open the smart portal. Providers can fill information/data in this portal and after submitting, this data was updated on the database.

\section{Blockchain Principals:}

Here are five basic principles underlying the technology.

1. Distributed Database:

Each party on a blockchain has access to the entire database and its consummate history. No single party controls the data or the information. Every party can verify the records of its transaction partners directly, without an intermediary.

2. Peer-to-Peer Transmission:

Communication occurs directly between peers instead of through a central node. Each node stores and forwards information to all other nodes.

\section{Transparency}

Every transaction and its associated value are visible to anyone with access to the system. Each node, or user, on a blockchain, has a unique 30-plus-character alphanumeric address that identifies it. Users can choose to remain anonymous or provide proof of their identity to others. Transactions occur between blockchain addresses

\section{Irreversibility of Records:}

Once a transaction is entered into the database and the accounts are updated, the records cannot be altered, because they're linked to every transaction record that came before them (hence the term "chain"). Various computational algorithms and approaches are deployed to ensure that the recording on the database is permanent, chronologically ordered, and available to all others on the network.

\section{Computational Logic:}

The digital nature of the ledger means that blockchain transactions can be tied to computational logic and in essence programmed. So users can set up algorithms and rules that automatically trigger transactions between nodes.

\section{IMPLEMENTATION}

\section{A. Smart Patient's Wallet Application:}

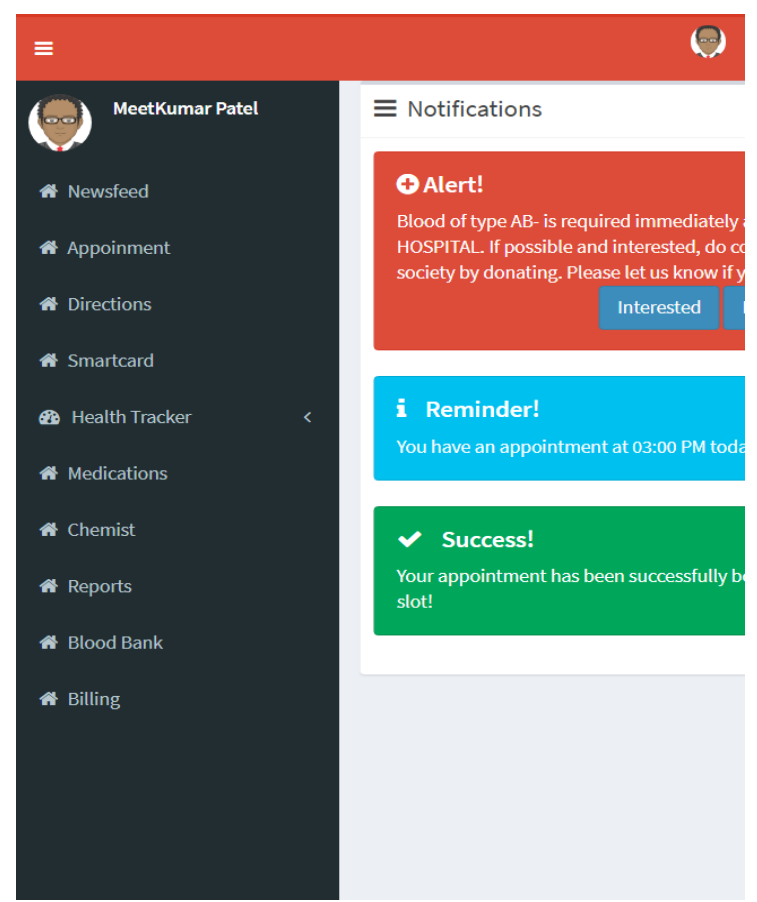

Fig 4 Smart Patient's Wallet Application

We used cross-platform approach to make smart patient's wallet application. For blockchain and store data we used hyperledger framework as backend along with PHP CodeIgniter framework. The patient application contains various facilities like appointment, medications, Health reports, Smart card, Health tracker, chemist and etc. The patient can manage and control their medical records using this application. The patient can also grant access to different providers 
using this application. The patient can view who is accessing their medical records. Also, there are including various functionalities for patient convenience.

\section{B. Providers Application:}

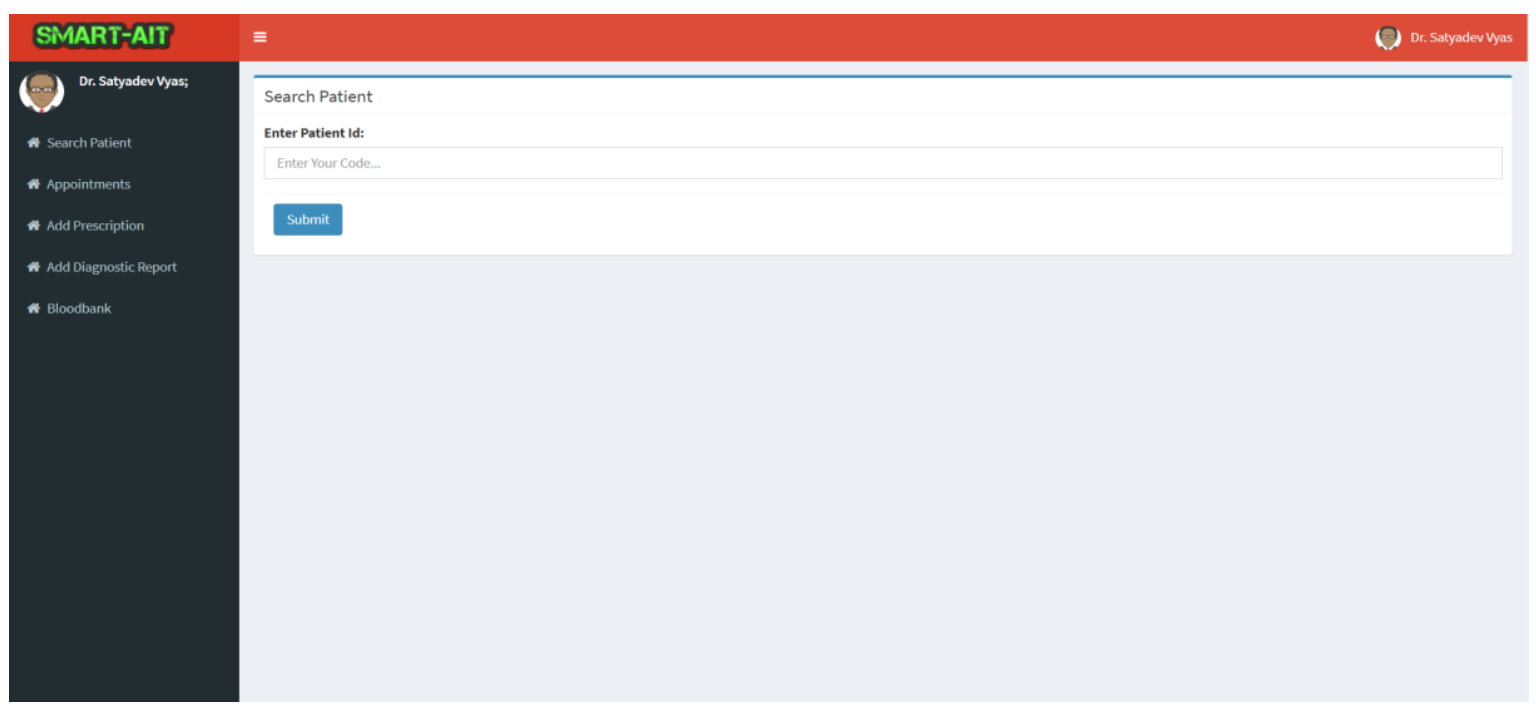

Fig 5 Smart Doctor Portal

In the figure, we see the doctor penal one of the provider. Here doctor can update and view the patient records if patient grants an access. The doctor can add prescription, add diagnostic report accordingly diseases. Here doctor can also scan QR code to access the patient information/medical records. This portal is totally web-based that's it is easily accessible to any devices with a web browser. We used MVC CodeIgniter framework and AdminLTE responsive layout for this portal.

\section{CONCLUSION}

In the era of patient-centered care, the prototype of smart health wallet offers patients improved control over their medical information and provide transparency between patients and medical data providers. The smart health wallet prototype provides a proof of concept system, demonstrating how principles of decentralization and blockchain architecture could contribute to secure patient's medical record and also gave full control over their medical records. We look forward to continued work on the smart health wallet also release our framework on GitHub as a platform for further development.

\section{REFERENCES}

[1] Mandl, Kenneth D., David Markwell, Rhona MacDonald, Peter Szolovits, and Isaac S. Kohane. "Public Standards and Patients' Control: how to keep electronic medical records accessible but private.” Bmj 322, no. 7281 (2001): 283-287

[2] Office of the National Coordinator for Health Information Technology. (2015). Report to Congress. "Report on Health Information Blocking." : https://www.healthit.gov/sites/default/files/reports/info_blocking_040915.pdf

[3] Nakamoto, Satoshi. "Bitcoin: A peer-to-peer electronic cash system." (2008).

[4] Gustavo H. M. B. Motta and Sergio S. Furuie "A Contextual RoleBased Access Control Authorization Model for Electronic Patient Record"

[5] https://www.media.mit.edu/research/groups/1454/medrec

[6] https://ethereum.org/

[7] http://hyperledger.org/

[8] https://en.wikipedia.org/wiki/Hyperledger

[9] https://blockchainhealth.co/

\section{BIOGRAPHY}

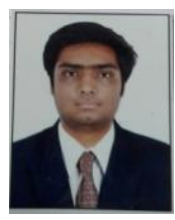

Meetkumar Patel is currently pursuing an Undergraduate degree in Computer Engineering from Ahmedabad Institute of Technology. He is interested in Machine Learning, Natural Language Processing, Programming Methodology and Computer Security. 